\title{
RAHMEN SCHAFFEN
}

\section{Liebe Leserin, lieber Leser,}

die Elektrifizierung des Antriebs ist das Kernthema der Fahrzeugentwicklung schlechthin. Sie ist auch notwendig. Daher ist es natürlich, dass sich die aktuelle ATZ in ihrem Leitthema ausführlich dieser Fragestellung widmet. Dabei geht es nicht nur um das technisch Machbare, sondern auch um das ökonomisch Sinnvolle und auch darum, was denn nun die Kunden wünschen oder akzeptieren. Auf die Frage, ab welchem Anteil regenerativer Energien am Strommix eine flächendeckende E-Mobilität aus ökologischer Sicht Sinn macht, wurde in vergangenen Editorials bereits ausführlich eingegangen. Mindestens genauso wichtig ist es, Kunden für das Elektroauto zu interessieren und ihnen Sicherheit zu geben hinsichtlich Zuverlässigkeit, Diagnoseund Reparaturfreundlichkeit dieser neuen Technik.

Im Prinzip befindet sich die Industrie am gleichen Punkt, an dem sie stand, als die Hybridtechnik vor einigen Jahren in den Markt eingeführt wurde. So kann es nicht verwundern, dass sich die Zahl der Besitzer von E-Autos aktuell eher in homöopathischen Größenordnungen bewegt. BMW beschäftigt sich ausführlich mit der Thematik des Technologiemarketings, und das ika setzt sich dezidiert mit den Kundenanforderungen auseinander.

Die nicht nur aus Kundensicht wichtige Frage, wie Fehler sicher erkannt und behoben werden können und wie beispielsweise im Rahmen der Hauptuntersuchung (HU) eine technikgerechte Überprüfung stattfinden kann, ist sicherlich ein erster Grund. Der VdTÜV beschäftigt sich daher in einer groß angelegten Studie mit den notwendigen Regularien für eine erforderliche Funktionsprüfung für Stecker, Ladeanschlüsse, Kabel und vor allem die Batterie von Elektrofahrzeugen. Die bis 2016 angelegte Untersuchung soll in eine HU-
Hochvoltrichtlinie für Elektroautos münden, die Kunden, Prüfern und Herstellern die notwendigen gültigen Rahmenbedingungen gibt.

Ein zweiter wichtiger Punkt ist die notwendige Infrastruktur. Viele potenzielle Kunden von Elektrofahrzeugen verfügen nicht über eine private Lademöglichkeit. Solange Kommunen, Städte und Unternehmen sich dieser Problematik nicht mit Nachdruck annehmen, wird daher die E-Mobilität eine Randerscheinung bleiben. Es mangelt allerorten an ganzheitlichen Konzepten zur Parkraumgestaltung und Verkehrsführung. Soll also der Wille zur Elektromobilität kein Lippenbekenntnis bleiben, ist hier schnelles und nachhaltiges Handeln vonnöten. Es sollten nun endlich die Rahmenbedingungen geschaffen werden, die eine nachhaltige Einführung von Elektromobilität erst ermöglichen.

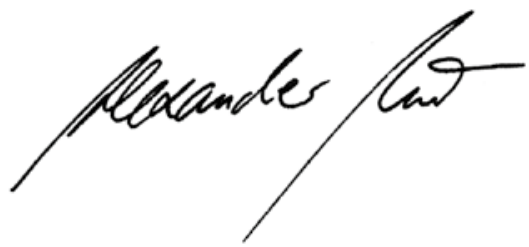

DR. ALEXANDER HEINTZEL, Chefredakteur Wiesbaden, 25. September 2014

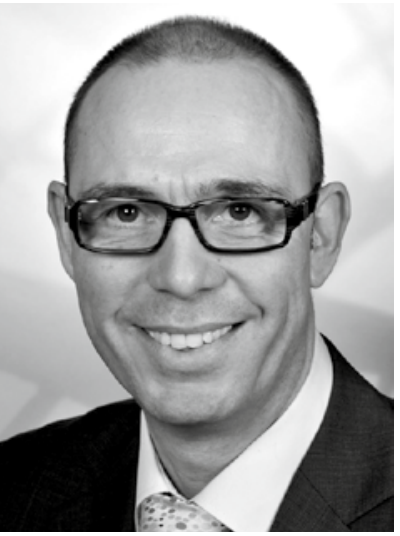

Wir entwickeln, was bewegt

IAV - Ihr Partner

für Automotive Engineering

- Antriebselektronik

- Cockpit

- Fahrwerk

- Getriebe

- Elektrifizierung Antriebsstrang

- Energieversorgung

- Exterieur und Sicherheit

- Integrale Fahrzeugfunktion

- Interieur

- Methoden und Tools

- Mobility

- Powertrain Integration

- Product Lifecycle

- Vehicle Electric/Electronic

- Verbrennungsmotor

Mehr zu unserer einzigartigen Kompetenzbreite erfahren Sie auf www.iav.com 